\title{
Intermédialités
}

Histoire et théorie des arts, des lettres et des techniques

Intermediality

History and Theory of the Arts, Literature and Technologies

\section{Cinéma, médiation et transmission chamaniques, d'après Poétique du cinéma de Raoul Ruiz}

\section{Marie-Hélène Mello}

Numéro 5, printemps 2005

Transmettre

Transmitting

URI : https://id.erudit.org/iderudit/1005494ar

DOI : https://doi.org/10.7202/1005494ar

Aller au sommaire du numéro

Éditeur(s)

Centre de recherche sur l'intermédialité

ISSN

1705-8546 (imprimé)

1920-3136 (numérique)

Découvrir la revue

Citer cet article

Mello, M.-H. (2005). Cinéma, médiation et transmission chamaniques, d'après Poétique du cinéma de Raoul Ruiz. Intermédialités / Intermediality, (5), 99-117. https://doi.org/10.7202/1005494ar
Résumé de l'article

Dans son essai « Pour un cinéma chamanique ", Raoul Ruiz met en valeur les affinités qu'entretient l'image cinématographique avec le chamanisme. Cet article éclaire la notion ruizienne de " cinéma chamanique » afin d'amorcer une réflexion sur le cinéma en tant que médium chamanique qui préserve des qualités médiatiques liées à la transmission orale. L'auteur y aborde le rôle de l'image dans l'oeuvre de Ruiz — associé à la mémoire, à l'art combinatoire et $\mathrm{au}$ " film potentiel » - de même que la conception du spectateur qui en découle. L'article se conclut par une analyse du Film à venir (Ruiz, 1997) d'après L'écoute filmique de Véronique Campan qui tente de déterminer ce que pourrait être le « caractère chamanique » du son au cinéma. 


\title{
Cinéma, médiation et transmission chamaniques, d'après Poétique du cinéma de Raoul Ruiz
}

\author{
Marie-Hélène Mello
}

aoul Ruiz est parmi les rares cinéastes à avoir associé cinéma et chama-
nisme dans le chapitre «Pour un cinéma chamanique» de sa Poétique du
cinémal. Sans porter directement sur la pratique chamanique des civilisations
primitives, l'essai se consacre à la figure du chaman pour mettre en valeur la
conception ruizienne de l'image cinématographique. En tant que spécialiste de
l'expérience extatique, psychopompe et médiateur ${ }^{2}$, le chaman devient une figure
du passeur qui renvoie à la transmission orale. Le passage chamanique - voyage
initiatique permettant l'entrée en contact avec l'«autre monde»- implique
l'apprentissage d'un langage secret et correspond à une expérience poétique.
Plusieurs recherches en ethnologie et en histoire des religions ${ }^{3}$ ont déjà su

1. Raoul Ruiz, Poétique du cinéma 1: miscellanées, Paris, Éditions Dis voir, 1995. Désormais, les références à cet ouvrage seront indiquées par le sigle « $\mathrm{PC}$ » suivi de la page et placées entre parenthèses dans le corps du texte.

2. Mircea Eliade, Le chamanisme et les techniques archaïques de l'extase, Paris, Éditions Payot, 1983 [1951]. Désormais, les références à cet ouvrage seront indiquées par le sigle «LC» suivi de la page et placées entre parenthèses dans le corps du texte.

3. Eliade considère l'euphorie préextatique du chaman comme une source du lyrisme universel: l'état second obtenu par le chaman «met en branle la création linguistique et les rythmes de la poésie lyrique »; "L'acte poétique le plus pur s'efforce de recréer le langage à partir d'une expérience intérieure qui, pareille en cela à l'extase ou à l'inspiration religieuse des "primitifs", révèle le fond même des choses. C'est à partir 
rendre compte de la proximité entre le poète et le chaman, mais Ruiz innove en associant le chamanisme au travail du cinéaste, puis à l'expérience spectatorielle. Par le biais des images, le cinéaste «témoigne» d'un voyage chamanique en créant un film ouvert et polysémique dans lequel «plonge» le spectateur, qui à son tour modifie le film d'après son expérience vécue et sa mémoire filmique. Le film est dès lors perçu comme un lieu d'échange et de rencontre qui suppose la coexistence de plusieurs temps et de plusieurs espaces, de même que la participation active du spectateur.

«Pour un cinéma chamanique» esquisse les affinités qu'entretient le cinéma avec la médiation et la transmission chamaniques en proposant certaines relations entre l'oralité et l'image, voire une oralité de l'image. La thématisation du cinéma chamanique, suivie de l'étude des caractéristiques fondatrices du cinéma proposé par Ruiz, serviront à montrer que le cinéma chamanique et la pratique du cinéaste-chaman dépeints dans l'essai sont liés à une réflexion sur le rapport qu'entretient le cinéma de fiction avec la médiation traditionnelle fondée sur l'oralité. Le court-métrage Le film à venir (Raoul Ruiz, 1997) et l'essai L'écoute filmique de Véronique Campan permettront, en dernier lieu, de compléter cette réflexion sur l'image en abordant le caractère «chamanique » du son au cinéma.

\section{LA POÉSIE, LE QUOTIDIEN OU LE FILM « FAIT MAIN »}

Dans «Pour un cinéma chamanique», Ruiz propose une nouvelle façon de filmer basée sur les «variétés de l'expérience du monde sensibles.» (PC, p. 80)

de créations linguistiques de cet ordre, rendues possibles par l'“inspiration” pré-extatique, que les "langages secrets" des mystiques et les langages allégoriques traditionnels se sont cristallisés plus tard. » (LC, p. 396-397) Dans «Shamanism and Poetry», E. V. Revunenkova qualifie de chaman-poète «celui qui fait vivre les légendes»: «The special status of the poet, who is at the same time a prophet and a seer, is identical to that of a priest or of a religious leader who acts as a mediator between the earth and the heavens. (E. V. Revunenkova, Shamanism: Past and Present, Budapest, Hungarian Academy of Science, 1989, vol. 2, p. 395)

4. Véronique Campan, L'écoute filmique :écho du son en image, Saint-Denis, Presses universitaires de Vincennes, 1999. Désormais, les références à cet ouvrage seront indiquées par le sigle «EF » suivi de la page et placées entre parenthèses dans le corps du texte.

5. Cette démarche nécessaire à la création d'un cinéma chamanique évoque la réflexion pasolinienne sur la langue de la «poésie naturelle de l'existence », qui « exprim[e], 
L'évolution du cinéma s'exprimerait d'après deux principales tendances: le naturalisme («approche industrielle») associé au travail des frères Lumière et comparé à la science; et l'artifice, « approche artisanale » issue de Méliès, qui s'apparente à la sorcellerie. À ces deux pôles du cinéma sont associés deux types de cinéastes et théoriciens: les filiationnistes et les irruptionnistes. Privilégiant l'expérimentation et l'exploration, les filiationnistes «tentent de se positionner par rapport aux origines lointaines du cinéma, avant de l'expliquer dans les termes des disciplines existantes », alors que les irruptionnistes « considèrent le cinéma comme un phénomène original et inattendu » et privilégient ce que Ruiz appelle les «pouvoirs magiques » et le «vertige» (PC, p. 73). Bien qu'il souligne que l'approche industrielle domine aujourd'hui, Ruiz s'intéresse manifestement davantage au travail des cinéastes irruptionnistes, qu'il compare à des chamans ${ }^{6}$. Ceux-ci produisent des films «faits main, faits maison, artisanaux» (PC, p. 75), leur démarche s'apparente aux vieux métiers et témoigne d'un esprit de bricolage.

Pour Ruiz, le cinéma chamanique consiste à «faire voyager [le spectateur] jusqu'aux confins de la création, avec la simple juxtaposition d'un petit nombre d'images frémissantes» (PC, p. 88). L'essai montre que le vertige du cinéma chamanique consiste à faire jaillir l'inattendu et l'indescriptible, la poésie du quotidien ${ }^{7}$ : par le contraste entre les images, les sauts insoupçonnés, les acci-

en les racontant, en les évoquant, des choses de la vie plus humbles et plus fortuites, ou bien ce que n'importe quelle vie contient, en abondance, de mystérieux ou d'irrationnel. » (Pier Paolo Pasolini, L'expérience hérétique. Langue et cinéma, trad. Anna Rocchi Pullberg, Paris, Éditions Payot, 1976 [1971], p. 12. Désormais, les références à cet ouvrage seront indiquées par le sigle « EH » suivi de la page et placées entre parenthèses dans le corps du texte)

6. D'après Ruiz, les films artisanaux ensorcellent, possèdent la faculté de ravir l'âme: «[...] en espagnol, pour ravir l'âme d'une personne, on dit hechizo, ce qui signifie aussi bien fait artificiel qu'ensorcellement.» (PC, p. 72)

7. Chez Ruiz comme chez Pasolini, «la réalité est du cinéma en nature ( $\mathrm{EH}$, p. 99), elle "parle par elle-même» à travers le cinéma. Si, d'après Pasolini, le cinéma est une langue potentielle hypothétique et, virtuellement, «un plan-séquence infini, comme la réalité qui peut être reproduite par une caméra invisible» (EH, p. 99), le cinéma chamanique de Ruiz consiste à révéler le devenir-cinéma des objets du quotidien, et à explorer les potentialités par des jeux combinatoires. Dans son texte, Ruiz fournit l'exemple d'une «activité cinématographique chamanique » consistant à filmer un abrégé des actions banales d'un homme durant une semaine, puis d'explorer les diverses séquences de montage possibles afin de recréer diverses combinaisons étranges mais bien réelles (PC, p. 76-77). 
dents magiques, le cinéaste-chaman révèle « les multiples mondes possibles qui coexistent près de chez nous » (PC, p. 88). Il est perçu comme un médiateur, car il évolue dans deux (ou plusieurs) mondes dont il permet la rencontre, la contamination à même le film. Réceptif et doté de la double vision ${ }^{8}$, il est également responsable d'assurer la transmission: sans toutefois raconter ou témoigner directement de son «voyage », son rôle est de créer un film ouvert qui permettra à son tour la rencontre du spectateur avec le monde autre. Le film chamanique est avant tout "poétique » en raison de cette ouverture et de son caractère imparfait, inachevé. Ce sont les failles de l'œuvre - décrites comme pistes de décollage et d'atterrissage - qui permettent la communion avec l'«autre monde ». Ainsi, bien plus qu'un messager ou un interprète, le cinéaste-chaman agit en tant que passeur; ce qu'il transmet est avant tout la faculté de voir, non un «message des esprits » comme le chaman traditionnel. Le film qu'il produit est à son tour milieu de rencontre et de communion. La création d'un film participe ainsi de l'expérience vécue et partagée, «pierre angulaire » du chamanisme ${ }^{9}$.

En tant que carrefour de plusieurs mondes et de milieu où le spectateur rencontre l' «au-delà de l'image», le film chamanique miserait sur ce qui est indescriptible par l'usage commun des mots, menant nécessairement à une certaine impasse de la critique. D’après Ruiz, le cinéaste-chaman doit accorder une extrême importance aux objets - intraduisibles en soi ${ }^{10}$ - qui composent le plan, au détriment des personnages: sa démarche consiste à saisir dynamiquement les objets de la réalité afin de créer des objets poétiques pour lesquels les règles nécessaires à la compréhension «demeurent uniques, toujours singulières et restent à découvrir par chacun. Elles ne sont descriptibles ni a priori, ni a posteriori » (PC, p. 75). Il n’existe donc ni «clé», ni témoignage possible de l'expérience chamanique - autre que par les images. Pour Ruiz, l’image vivrait

8. Dans son essai «Le cinéma comme voyage clandestin», Ruiz précise cette faculté de «double vision» du cinéaste-chaman: «ce qui permet de voir les choses de ce monde entourées de choses venues d'un autre monde, situé dans un autre lieu ou dans un autre temps. » (PC, p. 111)

9. Jean-Patrick Costa, Les chamans: hier et aujourd'hui, Paris, Éditions Flammarion, coll. «Dominos", 2001, p. 18.

10. La conception ruizienne de ces «objets poétiques » évoque le cinème pasolinien, unité minimale du cinéma : «Un objet de la réalité, pris en tant que cinème, est lui aussi, en soi, intraduisible: c'est un fragment brut de réalité.» (EH, p. 172) 
au-delà de la langue, car elle possède un langage secret et magique qui lui est propre. Si l'expérience chamanique traditionnelle consiste à prendre possession d'une langue nouvelle (LC, p. 89), celle du cinéaste-chaman ou du spectateur est essentiellement poétique et concerne la familiarisation avec un certain «langage des images».

Dans L'expérience hérétique, Pasolini insiste sur la «prégrammaticalité » de l'image poétique, caractéristique qui semble également proche de la conception ruizienne de l'image cinématographique: en tant que symboles du langage visuel, les objets ont déjà une longue histoire prégrammaticale qui les précède, tandis que leur histoire grammaticale est inventée ponctuellement. De même, le cinéma chamanique décrit par Ruiz est «capable d'inventer une nouvelle grammaire chaque fois qu'il passe d'un monde à l'autre» (PC, p. 80). Ainsi, le film chamanique n'échappe pas au langage, même s'il naît de ses failles. Ceci n'est pas sans rappeler la réflexion bazinienne à propos de la relation contradictoire qu'entretient le cinéma avec le langage ${ }^{11}$ : le cinéma ramène à la perception brute d'un monde avant le langage, avant la connaissance, tout en étant lui-même «langage du monde» (PC, p. 104). Christine Buci-Glucksmann et Fabrice Revault d'Allonnes distinguent la position de Ruiz de celle de Bazin:

[...] le cinématographe serait le moyen de rencontrer le monde d'après la connaissance: irrationnel, illogique, proche de l'in-conscient. Un monde où, au-delà du savoir (mieux encore: au sein même du savoir), persiste du mystère, de la sorcellerie. Un monde où persistent, par-delà l'accumulation d'images, le «déjà vu » et le «déjà filmé», du jamais vu, de l'incroyable, de l'émerveillement et de la terreur, du possible ${ }^{12}$.

Ruiz transcenderait donc la contradiction bazinienne. Pour lui, «le monde est grammatical ${ }^{13}$ ", tout est langage; il s'intéresse particulièrement à sa structure $^{14}$ qu'il met à l'épreuve pour voir ce qui reste.

11. Voir André Bazin, Qu'est-ce que le cinéma? I. Ontologie et langage, Paris, Cerf, coll. « $7^{\mathrm{e}}$ art », 1958 .

12. Christine Buci-Glucksmann, Fabrice Revault d'Allonnes, Raoul Ruiz, Paris, Éditions Dis voir, 1987, p. 80-81. Désormais, les références à cet ouvrage seront indiquées par le sigle «RR» suivi de la page et placées entre parenthèses dans le corps du texte.

13. Propos prêtés à Melvil Poupaud dans Les destins de Manoel (Raoul Ruiz, 1985).

14. L'omniprésence d'une réflexion théorique sur les processus du rêve et de la mémoire dans les films de Ruiz nous apparaît liée à sa volonté d'explorer les structures du «langage de la réalité ». Rêve et mémoire sont aussi les exemples fournis par Pasolini 


\section{Le SPECTATEuR-Voyageur et Le PAsSAge}

Ruiz associe le cinéma au voyage, au passage magique vers l'autre-monde, évoquant le rôle traditionnel du chaman dans les sociétés primitives: il confère au cinéaste les fonctions de psychopompe, de médiateur, de passeur et de poète décrites par Mircea Eliade. Aux deux tendances du cinéma (industrielle, artisanale) qu'il oppose correspondent deux types de voyage du spectateur: alors que l'approche industrielle crée le voyage organisé, dans lequel «le plaisir vient du respect sadique du programme ${ }^{15}$ ", l'approche artisanale vise le vrai voyage, expérience unique caractérisée par les «accidents magiques, les changements d'avis, les découvertes, les émerveillements inexplicables et le temps perdu, et retrouvé à la fin du film» (PC, p. 76). Dans ses films, Ruiz a lui-même recours à plusieurs types $\mathrm{d}^{\prime}$ ' accidents » volontaires, trucages visibles qui déstabilisent et remettent en question la position du spectateur ${ }^{16}$; par exemple, une bouche au mouvement légèrement décalé par rapport à la voix qui lui est prêtée.

Créée de façon artisanale et saturée d'objets poétiques intraduisibles, c'est l'image qui est responsable de ce voyage temporel et spatial décrit par Ruiz. L'agencement des images d'après l'art combinatoire ${ }^{17}$ permet de produire des

pour exprimer que «la communication visuelle qui est à la base du langage cinématographique est $[\ldots]$ extrêmement brute, presque sauvage» et que «l'instrument linguistique sur lequel se fonde le cinéma est [...] de type irrationnel» (EH, p. 137).

15. Le réalisateur critique ici la méthode hollywoodienne, qui fait fi du travail du spectateur et le condamne à suivre une ligne droite dont l'issue est généralement prévisible (à ce sujet, voir «Théorie du conflit central», PC, p. 9-23). Ce cinéma s'opposerait à la liberté et à l'ouverture visées par le cinéma chamanique.

16. Buci-Glucksmann et Revault d'Allonnes soulignent cette tendance - associée à la pratique artisanale dans «Pour un cinéma chamanique » — chez Ruiz : «Jamais Ruiz ne recherche seulement l'effet pour l'effet, l'illusion pour la seule séduction; il donne toujours à voir en même temps qu'il y a effet, procédé, trucage. Il souligne, désigne qu'il y a illusion. » Cette démarche pointant le «faux » conduit paradoxalement, selon les deux essayistes, à une «œuvre de vérité »: «Tout le cinéma ruizien est l'exploration de cette trahison, [celle-ci] débouche sur une autre "réconciliation" de l'image avec... un autre réel. » (RR, p. 44)

17. Diverses techniques sont proposées par Ruiz pour favoriser le vrai voyage: la production de «monstres» par le biais de l'art combinatoire (PC, p. 77), la technique «Il ponte» qui consiste à avoir recours à des agents anamorphiques (p. 80), et l'emploi d'images-situations: «L'image-situation est l'instrument qui permet l'évocation et l'invocation des êtres imagés. C'est elle qui sert de pont, d'aéroport, aux multiples films qui vont coexister dans le film que l'on verra.» (p. 111) 
«séquences rituelles» qui font «voyager dans un au-delà où habitent les fantômes du temps perdu» (PC, p. 77). Les pouvoirs conférés à l'image cinématographique permettent ainsi d'entrevoir un rapprochement avec les civilisations orales, où «le "médium” est un lieu dans lequel on baigne », où «l'accent n’est pas mis sur ce qui est "séparé" et que le médium doit réunir, mais sur la processualité de la médiation en tant que performance ${ }^{18} »$. L'exemple fourni par Ruiz des préceptes du peintre Shih-T'ao l'illustre bien: «Nous pénétrons la peinture. La pluralité des événements devient un tout organique auquel nos yeux et nous-mêmes appartenons. » (PC, p. 86) C'est ainsi que l'image entraîne la plongée dans l'autre monde, devient à la fois lieu de passage et milieu de rencontre, suggérant une proximité avec la médiation traditionnelle fondée sur la transmission orale. La spectature est dès lors perçue comme un rituel, une cérémonie.

Le «vrai voyage» décrit par Ruiz pourrait être lu comme une définition de l'enchantement au cinéma, l'apparition de «la patine de ce qui n'en a pas » (SAS, p. 29). L'«ensorcellement» du spectateur (analogue à la transe extatique du chaman) a part liée avec le rêve, mais aussi avec la «mémoire vivante»:

[...] celui qui s'est endormi réellement, ou rituellement, fait partie du film en ceci que désormais il n'assiste pas seulement à l'atterrissage des images et des événements, mais à leur décollage, et ces images s'envolent, tantôt en direction du film, tantôt vers le spectateur lui-même à la recherche de ses multiples vies privées. (PC, p. 115)

Cette définition de la communion du spectateur avec le film - qui lie ensemble, fait coexister ses multiples vies passées et possibles - évoque la structure multiple de Trois vies et une seule mort (Raoul Ruiz, 1996) et les nombreuses vies du personnage incarné par Marcello Mastroianni. Mais l'enchantement du spectateur provoque également un sentiment d'extase : «Le passage est l'élément de surprise qui procure non seulement une soudaine illumination, mais aussi le plaisir [...] l'intérêt réside moins dans les trajets eux-mêmes que dans la beauté du saut d'un monde à l'autre.» (PC, p. 80) Ce saut est une expérience esthétique de communion avec l'au-delà de l'image, qui évoque aussi la traversée du chaman:

18. Johanne Villeneuve, «La symphonie-histoire d'Alfred Schnittke. Intermédialité, cinéma, musique », Intermédialités, $\mathrm{n}^{0}$ 2, «Raconter», automne 2003, p. 14. Désormais, les références à cet article seront indiquées par le sigle «SAS » suivi de la page et placées entre parenthèses dans le corps du texte. 
[...] quand le shaman traverse du côté des morts en chantant «avec la voix des anciens ", il devient passeur, médiateur, mais il ne transgresse rien et ne provoque aucun scandale. [...] Comme la fluidité de la voix qui le guide, dans l'avènement de cette voix qui n'est plus tout à fait sienne, le shaman se coule dans le milieu où les anciens rencontrent les vivants. La performance réactive alors la voix des anciens comme ce qui est toujours déjà là, dans une sorte de latence collective: toute séparation $[. .$.$] est ainsi vécue au quotidien, parce que le legs des anciens pèse$ toujours de son poids; les dieux sont toujours présents, tandis que le passé a la consistance du présent.» (SAS, p. 14)

Ainsi, l'image telle que la conçoit Ruiz possède des pouvoirs d'enchantement similaires à la voix; sa réflexion conduit à penser l'oralité de l'image.

En plus de ravir l'âme, de s'ouvrir afin de permettre le passage et de 106 transmettre la faculté de la «double vision », le film chamanique offre la "possibilité de transmettre un monde privé en temps présent où siègent plusieurs passés et avenirs » (PC, p. 117). Ce passage à un autre mode de temps (la coexistence de temps multiples) pourrait être comparé, quoique inadéquatement, à celui de l'hypnose ou du rêve. Dans Film, perception et mémoire, Jean-Pierre Esquenazi décrit l'enchantement du spectateur en termes de "passage d'un corps à l'autre», de «substitution de corps imaginaires»:

[...] c'est à l'exploration de sa propre place à l'intérieur du film que le spectateur est livré. C'est dans la succession contradictoire, vacillante, fluctuante, de ses présences dans le film que le spectateur prend corps. Tout comme si l'acte du film consistait en la production d'un autre corps, d'un corps imaginaire [...] qui va devenir le corps du spectateur du film ${ }^{19}$.

Voyage à dimension temporelle donc, mais où le corps ne s'efface pas, n'est pas oublié: Ruiz s'oppose en ce sens à la conception du spectateur de Paul Virilio $^{20}$. La médiation corporelle du spectateur décrite par Esquenazi, bien que

19. Jean-Pierre Esquenazi, Film, perception, mémoire, Paris, Éditions de L'Harmattan, coll. «Logiques sociales", 1994, p. 105.

20. Dans La machine de vision (Paris, Éditions Galilée, coll. «L'espace critique», 1988), Paul Virilio souligne que, dans la salle de cinéma, les corps s'effacent et que le cinéma se passe du corps de l'artiste, sa matière première étant la lumière. Il est intéressant de noter que, même si l'artifice occupe une grande place dans leurs réflexions sur le médium cinématographique, Ruiz est loin d’y attribuer des conséquences aussi dramatiques. Malgré tout, l'essai de Virilio s'attarde à quelques aspects que nous pourrions associer aux traces d'oralité au cinéma ou au chamanisme tel qu'entendu par Ruiz: la lumière a la capacité de faire réagir «comme quand nous écoutons de la musique » 
non énoncée dans le texte de Ruiz - mais peut-être suggérée par l’idée de «voyage» - , est mise en fiction dans plusieurs de ses films, notamment Les trois couronnes du matelot (1983) et Généalogies d'un crime (1997), et participe au rôle de médiateur-chaman attribué au spectateur.

Selon Ruiz, le cinéma chamanique joue sur le «pouvoir de faire resurgir, ou d'évoquer, d'autres moments derrière les images que l'on voit» (PC, p. 77). Chaque film est multiple par essence: chaque image peut à elle seule contenir un ou plusieurs autres films. Le film auquel nous assistons est peuplé de spectres et constitue "un appel lancé à nos ancêtres, lesquels viennent, enveloppés dans leur invisible pellicule» (PC, p. 79). Ainsi, plus qu'une relation intersubjective entre le spectateur et le film (ou le spectateur et le cinéaste), il est question ici d'un rendez-vous avec l'au-delà de l'image. Ruiz rappelle que le perceptible n'est qu'une infime partie de la réalité, base de la croyance chamanique. Le terme d' «au-delà», quoique pratique, apparaît néanmoins imparfait: en termes chamaniques, ce qui est perceptible et ce qui ne l'est pas coexistent, sont indissociables. Mircea Eliade rappelle que les images mythiques associées au passage du chaman (le pont, la barque, l'échelle, le vol magique) «expriment la nécessité de transcender les contraires, d'abolir la polarité qui caractérise la condition humaine» (LC, p. 378). Par conséquent, il n'y a pas réellement d' "autre monde », mais essentiellement médiation entre le visible et l'invisible; c'est sur cet aspect «orphique » qu'insiste également Ruiz dans son essai.

\section{L'image: De LA MÉmoire Au FILM POTENTIEL}

La réflexion de Ruiz est largement axée sur la spectature perçue comme un travail de mémoire: tout spectateur possède une «banque » d'unités de mémoire (de faits vécus, ou d'autres films). La mémoire juxtapose les unités qui n'étaient pas liées a priori, et les combinaisons possibles sont infinies. Le cinéma chamanique est, à cet égard, un art combinatoire auquel participe le spectateur : «Tous, nous possédons un nombre colossal de séquences filmiques potentielles qui coexistent dans un espace ténu et dans un temps très bref. Ces séquences sont interchangeables et se superposent. Tous ces films dorment en

(Paul Virilio, La machine de vision, p. 63); la relation entre le corps et l'univers se trouve aujourd'hui modifiée, passage (selon les termes de Heidegger) du corps habitant l'univers au corps habité par l'univers (Paul Virilio, La machine de vision, p. 65). 
nous. » (PC, p. 77) L'expérience filmique est si bien intégrée à l'expérience vécue que le spectateur en vient à croire qu'il se souvient d'événements qu'il n’a pas expérimentés à l'extérieur du film. Sylviane Agacinski l'évoque dans Le passeur de temps:

Nos représentations mentales se nourrissent d'images et des choses vues, comme nos monologues intérieurs imitent silencieusement le discours. [...] Photographie et cinéma fabriquent des images qui s'incorporent à notre pensée. L'image indicielle ne bouleverse pas seulement notre expérience visuelle et nos moyens de connaissance, elle transforme également notre mémoire ${ }^{21}$.

La mémoire fabriquée du cinéma est ainsi reliée à nos propres souvenirs, qui, comme l'écrit Ruiz, «maintenant se lèvent et marchent vers nous comme les morts vivants d'un film d'horreur» (PC, p. 78). La réflexion d'Agacinski met en lumière la proximité entre ce processus qui découle des images et celui du discours. D’après Ruiz, «c'est l'image qui détermine la narration et non l'inverse» (PC, p. 108). La nouvelle forme de narration cinématographique chamanique qu'il souhaite développer a partie liée avec la musique, aux images qui fonctionneraient sur un «mode musical». Il fournit l'exemple du poème symphonique, dont la technique vise à «développer des figures aux valeurs purement musicales » (PC, p. 106), à imiter les bruits de la nature pour produire une «matière musicale» qui est, à la fois, thème et développement.

La logique combinatoire du film s'organise d'après des obsessions - ou thèmes incantatoires - pour produire des émotions poétiques: polysémiques, les images deviennent alors à la fois évocatoires et invocatoires:

Évocation mécanique d'événements qui ont déjà eu lieu ou qui arriveront, qui appartiennent à d'autres mondes même si ces mondes sont eux-mêmes des films, des dieux déjà morts ou d'autres à venir. Invocation des événements éternels ( $c f$. Whitehead): perpétuelle recréation en constant état de régénération ou de défaillance. Dans ce commerce avec l'au-delà, le film nous invite à un voyage le long d'un fleuve souterrain, tandis que depuis notre navire nous envisageons des figures issues de l'autre monde. (PC, p. 109-110)

Le travail de mémoire ${ }^{22}$ auquel convie le film chamanique - en tant que carrefour de déjà vu, de déjà filmé, de vies et de films possibles ou à venir —

21. Sylviane Agacinski, Le passeur de temps: modernité et nostalgie, Paris, Éditions du Seuil, 2000, coll. «La librairie du $x^{e}$ siècle», p. 109 (nous soulignons).

22. La perception de l'image présente ici d'étonnantes similitudes avec le travail de mémoire qui découle de l'œuvre d'Alfred Schnittke, d'après Johanne Villeneuve: 
implique donc un constant va-et-vient entre l'image cinématographique et la mémoire, qui se contaminent. En raison des multiples ponts qui se tissent entre le film et lui, le spectateur n'est pas récepteur d'un quelconque message unidirectionnel, ni même d'une histoire fixée d'avance, mais prend part à un processus circulaire en devenir. Néanmoins, s'il y a transmission, c'est bien celle de l'expérience de la coprésence des temps et des lieux; le film chamanique permet à la fois de rendre la mémoire présente et d'engendrer de nouvelles histoires.

Ce processus conduit Ruiz à qualifier le film chamanique de champ de mines: «En explosant il provoque des réactions en chaîne au sein de ces séquences filmiques et permet de produire certains événements. ( PC, p. 77) La structure du film chamanique est donc dynamique, instable, en constante métamorphose. Selon le cinéaste, tout film renferme nombre de films potentiels (ou films secrets) situés dans l'écart et jaillissant des «accidents».

Je veux juste faciliter le saut vers ce monde d'images qu'on appelle un film à l'intérieur duquel coexistent, simultanément, plusieurs autres films qu'au lieu d'ignorer, je cherche à rendre visibles autant qu'il est possible. J'évoque un cinéma qui renonce à sa capacité narrative, hypnotique, à sa puissance de ravissement et préfère se tourner lui-même dans le but de laisser proliférer des séries d'images circulaires, des hors-champs, qui profitent du déjà vu, tout ceci afin de pluraliser des séquences narratives, lesquelles se révèlent capables de donner naissance à une forme inédite de narration cinématographique, avec des règles à inventer, une poétique à découvrir. (PC, p. 107)

Ainsi, le film chamanique est une œuvre ouverte et polymorphe comme le système de pensée du chamanisme, un milieu où se rencontrent, se croisent et s'altèrent tous les films potentiels; et ce, grâce à la participation active du spectateur. Basé sur ces principes - ouverture, polymorphisme, liberté du spectateur - le cinéma chamanique est ainsi capable de restituer la multiplicité des mondes et de servir de pont entre le visible et l'invisible.

« [...] on y reconnaît un travail de mémoire foncièrement intermédial qui, par couches successives, conduit au devenir anachronique de l'œuvre. De larges pans sonores avancent et se retirent, se cristallisent et se désintègrent, ponctués de petites scènes absorbées à leur tour par les couches surdimensionnées du temps. » (SAS, p. 19) Ici, il y aurait plutôt lieu de parler de "pans d'images ", qui évoquent et invoquent, apparaissent et disparaissent, assurant le «lien vivant» entre plusieurs couches du temps. 


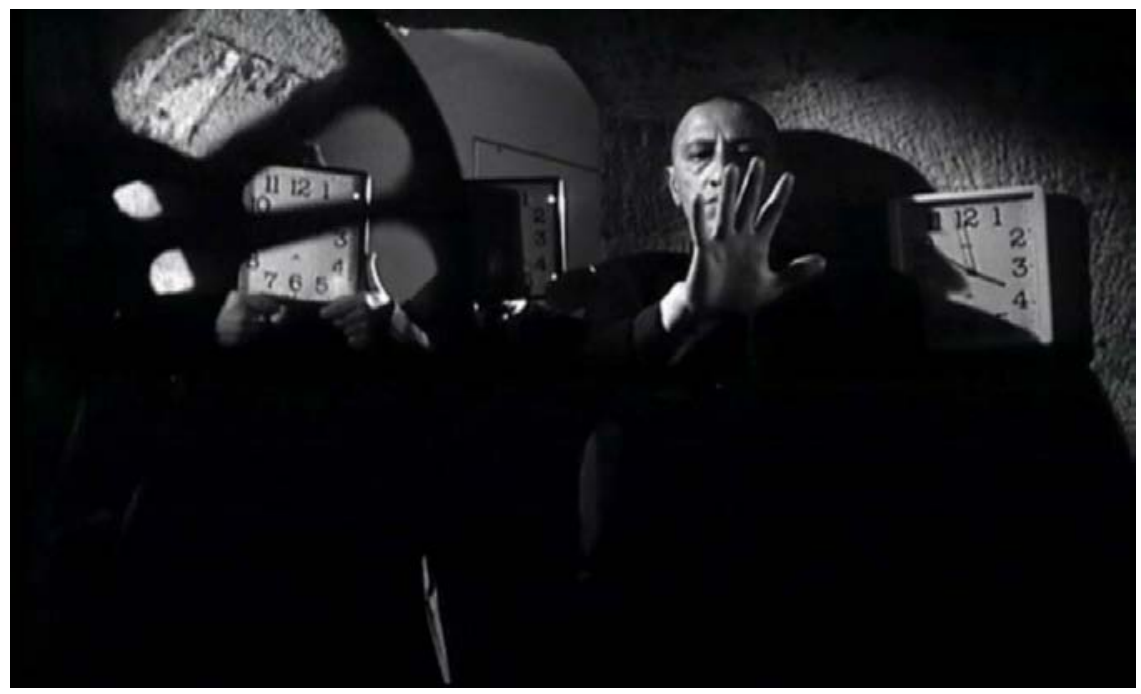

Fig. 1. La chambre des horloges (c) Raoul Ruiz, Le film à venir, 1997).

\section{LE FILM À VENIR}

Si les films de Ruiz apparaissent tous porteurs des questions centrales discutées dans «Pour un cinéma chamanique », Le film à venir, court-métrage réalisé en 1997, semble basé sur les principes mêmes qui conduisent le cinéaste à penser le cinéma en termes de chamanisme. Il s'agit d'un film qui, tout en possédant lui-même les caractéristiques du film chamanique relevées dans l'essai, pourrait être perçu comme une fable théorique à propos du cinéma chamanique.

Les Philokinètes, membres d'une société secrète de spectateurs qui pensent le mouvement et lui vouent un culte, étudient un fragment «hypnotique» de 23 secondes mis en boucle qu'ils appellent le «film à venir». Le court-métrage de Ruiz débute lui aussi avec une mise en boucle : la répétition d'une série de quatre plans fixes représentant des immeubles. Ce procédé - qui peut passer inaperçu au premier visionnement et constitue un jeu sur la puissance de ravissement du cinéma - fait en sorte que nous sommes d'emblée convoqués, nous aussi, en tant que spectateurs du «film à venir ». Le redoublement du titre du film dans le film n'est pas étranger à ce principe, mise en abyme toujours présente dans les films de Ruiz. Le réseau de fils électriques, de même que l'avion qui traverse le ciel, évoque également le voyage qui débute. 
La projection permanente du «film éternel» (qui n’est jamais montré à l'écran) se déroule dans une salle sacrée nommée «la chambre des horloges », où tous les temps coexistent. Le temps ne s'arrête pas, mais chaque personnage tient une horloge qui montre une heure différente des autres (fig. 1); les aiguilles ne tournent pas à la même vitesse et certaines horloges ne disposent pas d'aiguilles. Assister au film fait basculer dans un autre monde temporel, ce qui évoque le voyage temporel qui fait partie intégrante de la spectature dans l'essai de Ruiz.

La répétition rituelle du même fragment de film permet aux Philokinètes d'accéder à un état hypnotique et de parvenir à voir, même si, nous dit-on, «au début on ne voit rien». C'est par l'initiation que les membres accèdent à la transe chamanique, état de réceptivité au monde autre. Cependant, à maintes reprises, Ruiz joue avec la croyance du spectateur, le tire délibérément de son «hypnotisme» pour lui en faire prendre conscience, marquer la faille; c'est sans doute pour cette raison que le narrateur change à deux reprises, notamment lorsqu'on nous dit que la société de spectateurs traverse une «crise d'identité ». La voix dans les films de Ruiz participe toujours d'un «art du faire croire» tout en «montrant du vide»; elle a "une fonction altérante [...] qui délinéarise et multiplie» (RR, p. 27). Tout en faisant croire, elle semble demander «à quoi croit-on?", question à laquelle répondent Christine Buci-Glucksmann et Fabrice Revault d'Allonnes: «À un corps présent-absent, à cette oralité d'un Welles ou d'un Godard, à celle des cultures latino-américaines où le récit transmis et retransmis construit quelque chose qui manque à l'image: un corps de mémoire qui matérialise l'absence.» (RR, p. 27) La voix entretient donc un rapport étroit avec la nature fantomatique des images chez Ruiz, leur caractère évanescent, fragile et passager. Chez Ruiz, la dissonance ou la ressemblance entre ce qui est vu et ce qui est dit devient interne à l'image. Ce véritable «chassé-croisé de corps de langages » (RR, p. 26) mène à l'incertitude, participe à l'ouverture du film.

Les membres de la secte étudient aussi le «double-livre des arcanes dansantes", livre que l'on lit sans lire, allégorie du cinéma ${ }^{23}$. En effet, les prêtres ont fait «vœu d'illettrisme» et se contentent de feuilleter le livre composé de visages et de paysages de rêve, ce qui provoque «un mouvement vivace et

23. Dans son essai, Ruiz critique l'«approche industrielle» qui considère le cinéma comme un «livre à l'usage de qui ne sait pas lire» (PC, p. 71). Or, dans Le film à venir, les lecteurs du livre d'images doivent participer à un long processus d'initiation afin de parvenir à voir. 


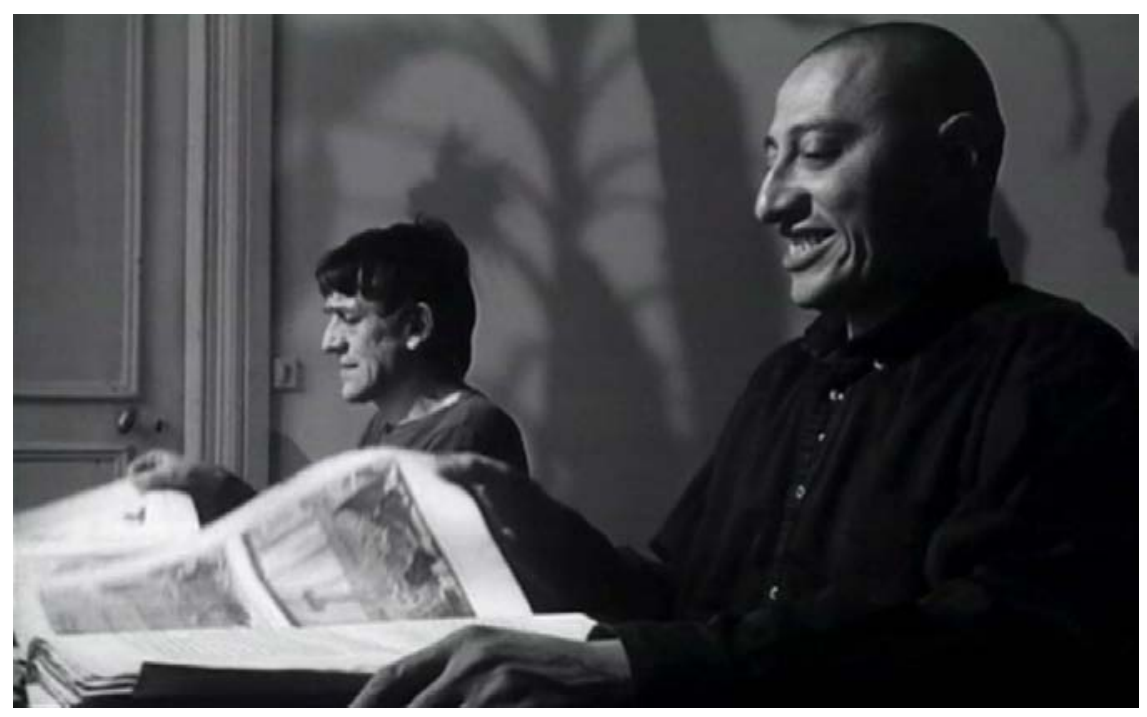

Fig. 2. Les prêtres philokinètes lisant le «double-livre des arcanes dansantes » (C) Raoul Ruiz, Le film à venir, 1997).

sublime» (fig. 2). Les images de ce livre nous sont montrées à l'aide du même procédé de mise en boucle employé au début du court-métrage. Le titre du livre, Mémoires, est très révélateur : il fait écho au «tout petit retour à soi » que provoque chaque boucle du film à venir, à «tous ces films qui dorment en nous », tel que l'écrit Ruiz dans «Pour un cinéma chamanique».

Les Philokinètes partagent la foi en le film à venir, c'est-à-dire «la conviction que le cinéma a une vie indépendante», qu'il est le «souffle primal d'une forme de vie nouvelle». Pour Ruiz, le cinéma est associé à la présence vivante, mais constitue également un monde autre qui a partie liée avec la mort. Le film ouvert et le caractère passager de l'image seraient liés au thème du mort-vivant:

L'image ressemble à ces fenêtres et portes cadrées sur du vide et qui ouvrent sur un autre film [...] Pure image de passage et de frontière, elle déstabilise tout réel. Le peuple des images cinématographiques ressemble comme un frère à tous les mortsvivants du cinéma ruizien. (RR, p. 14) 
Manifestation de l'entre-deux ontologique chez Ruiz, qui exploite les paradoxes inhérents à l’image et souligne la coexistence de la vie et de la mort dans l'autre monde ${ }^{24}$.

Lorsque le personnage principal «parvient à voir », il est fasciné, ensorcelé. L'enchantement est décrit comme «l'acte libérateur, l'euphorie délicieuse et sans douleur, l’immobile attente de la mort». De minuscules crânes apparaissent sur son $\operatorname{corps}^{25}$, puis son ombre se détache de lui (fig. 3-4); il devient un corps projeté. Tel le chaman durant ses transes extatiques, l'âme quitte le corps; c'est ce que révèle le narrateur alors que sont montrés les fils électriques du début du film. Tout en marquant notre entrée dans le film de Ruiz, ce réseau symbolise aussi le retour à l'origine de la mémoire cinématographique du protagoniste (en l'occurrence, le visionnement de Zorro dans son village natal). Il apparaît également renvoyer au labyrinthe des films possibles, au système à histoires multiples qui se chevauchent, susceptibles d'engendrer de nouvelles histoires ${ }^{26}$ que propose Ruiz dans son essai.

Le moment où le personnage voyage jusqu'à la première projection à laquelle il a assisté coïncide aussi avec les retrouvailles d'Aline, sa fille morte mais toujours vivante dans le «film à venir ». C'est une autre mémoire qui est convoquée ici, celle de l'expérience vécue, l'appel aux «fantômes du temps passé ». Le narrateur comprend alors qu'il est lui-même devenu une partie du «film à venir », conclusion qui semble liée en partie à sa participation à l'expérience filmique - façon d'évoquer assez littéralement la «plongée dans le film» et

24. Cette conception renvoie aussi à l'absence du principe de contradiction dans les cultures orales et à la «transcendance des contraires » que symbolise le chaman : «La culture orale induit une conception de l'expérience comme plénitude, densité, pour laquelle le principe de contradiction demeure inconnu; aussi les temps fusionnent-ils, de même que les espaces parcourus, dans l'unique creuset d'une collectivité en devenir de mémoire. » (SAS, p. 24)

25. Ce trucage qui produit un effet ludique pourrait faire référence au costume chamanique traditionnel : «En s'efforçant d'imiter le squelette [...], le costume chamanique proclame le statut spécial de celui qui le revêt, c'est-à-dire de quelqu'un qui est mort et est ressuscité. » (LC, p. 138)

26. D'autres films de Ruiz, par exemple Les trois couronnes du matelot, La ville des pirates (1984) et Trois vies et une seule mort, possèdent également une structure labyrinthique obéissant à ce principe «chamanique»: au contact les unes des autres, les histoires de base - déjà multiples - de chacun de ces films se multiplient davantage et créent des réseaux d'interprétation qui tendent vers l'infini. 


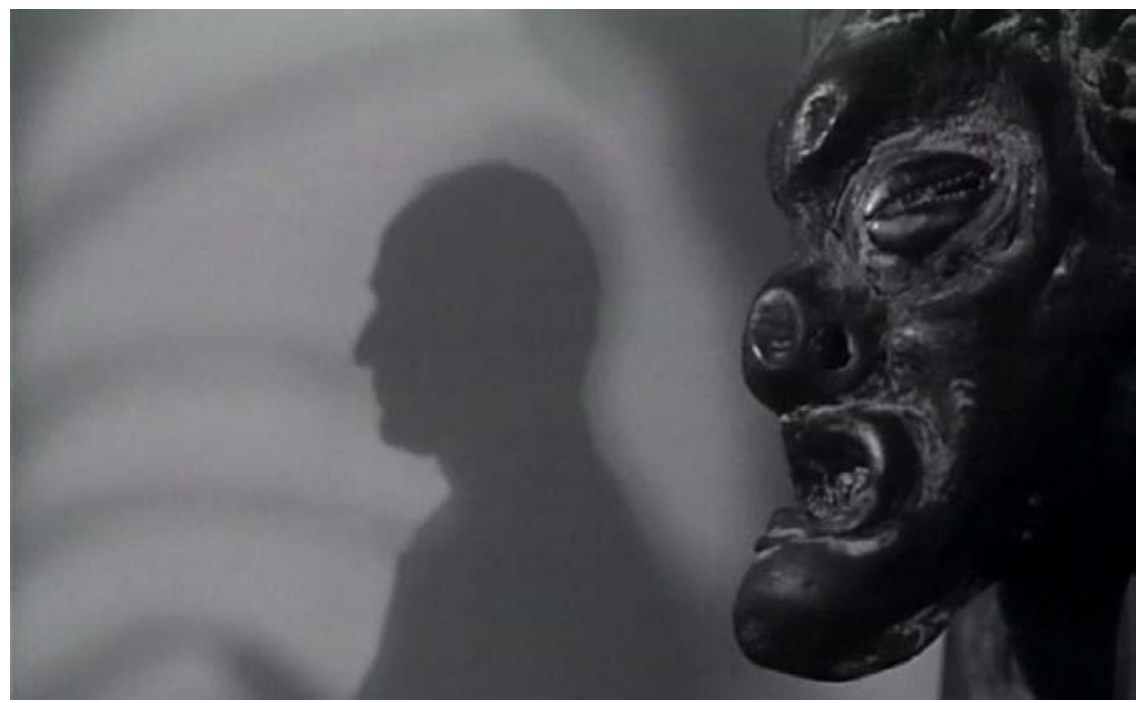

Fig. 3. L'enchantement ( Raoul Ruiz, Le film à venir, 1997).

d'insister sur le travail de mémoire du spectateur - et pourrait symboliser la substitution d'un corps imaginaire. Mais, par ailleurs, en raison du fondu au noir de la fin, tout ceci suggère que le personnage et le court-métrage entier font désormais partie de «notre» film à venir et de notre mémoire filmique.

\section{L'ÉCoute filmique, La tRACe et le chamanisme}

$\mathrm{Si}$ «Pour un cinéma chamanique» ne faisait pas état de l'aspect sonore de la spectature, Le film à venir fournit quelques exemples où le son participe à l'ouverture du film. Rappelons que la voix chez Ruiz - qu'elle soit conjointe ou disjointe de l'image, la précédant ou la commentant - participe aussi au paradoxe de l'«entre-deux ontologique» ruizien; elle crée un «corps de mémoire », fournit une voix à l'absence, participe au motif du «mort-vivant». Elle ouvre à une pluralité de sens, crée un effet de suspension et laisse planer l'incertitude. De même, la source et le rôle des divers bruits dans le film n'est jamais clairement identifiable: quand les deux prêtres philokinètes parcourent le double-livre, nous entendons un bruit évoquant les rebonds d'une balle de plastique et plusieurs craquements énigmatiques maintes fois répétés et mis en valeur dans la bande sonore. Dans la salle des horloges, une voix enregistrée et distortionnée répète «il est 17 heures 17 minutes, il est 17 heures, il est, il est, 


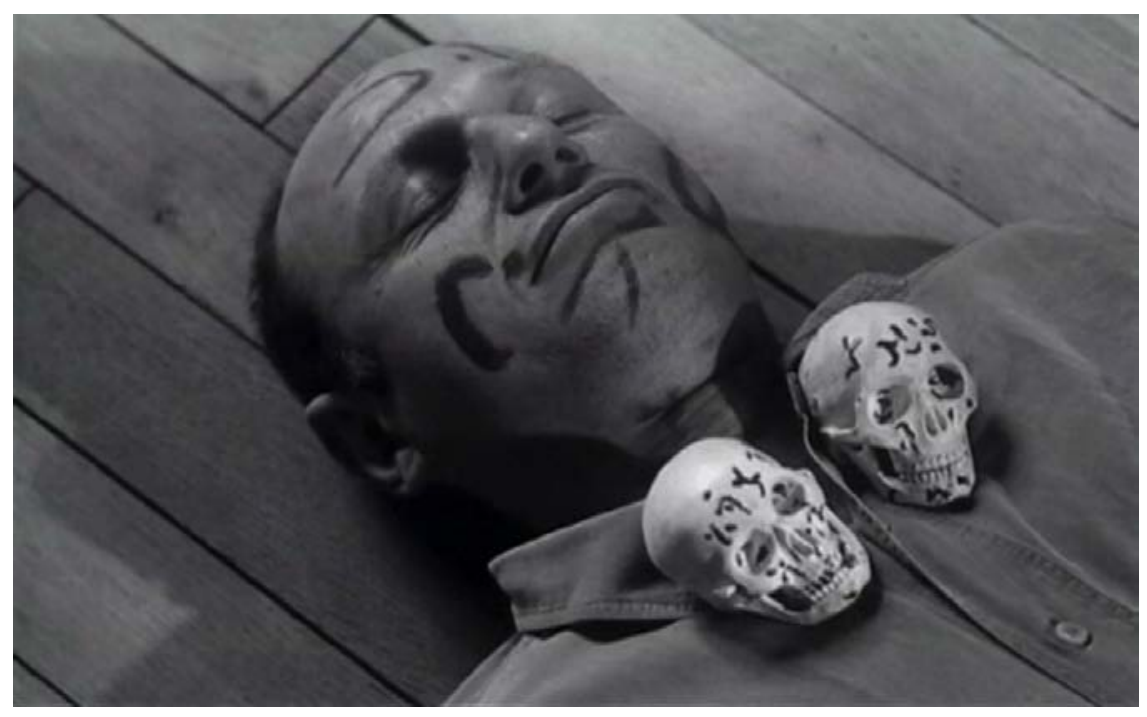

Fig. 4. L'apparition des crânes (@ Raoul Ruiz, Le film à venir, 1997).

il, il...» jusqu'à devenir un bruit diffus et obsédant. Impossible d'interpréter ces sons avec certitude: nous sommes laissés à nos impressions (une balle de plastique?), à nos hypothèses jamais vérifiables. La seule constante repérable est la répétition de trois types de sons délibérément mise en valeur. Il semblerait que le son participe au Film à venir, car il fait lui aussi appel à la mémoire du spectateur et il empêche de fixer le sens.

L'écoute filmique, essai de Véronique Campan axé sur la perception sonore au sein de la spectature, évoque la façon dont le son pourrait également participer au cinéma chamanique. S'appuyant sur la théorie de la perception de Husserl, Campan fonde sa réflexion sur l'impossibilité de saisir les objets de façon définitive, leur compréhension étant progressive et infinie. Liée au postulat ruizien du «film à venir», la perception est «un mouvement qui n'a pas de terme» (EF, p. 15), une série ouverte dans laquelle se côtoient les profils passés et les profils à venir de l'objet. Écouter un film, c'est «suivre les sons à la trace »: comme le film chamanique (et le nombre infini de films potentiels qu'il renferme), «le perçu n'a de chair, de densité, qu'en raison des multiples profils, latents, qu'il retient en lui» (EF, p. 16). C'est précisément sur ces «profils latents » que Ruiz cherche à attirer l'attention, dans son texte comme dans son court-métrage. D’après Campan, la trace atteste une présence tout en 
marquant la distance entre deux mondes; elle «troue le tissu représentatif » et est le vestige d'un objet «ici, maintenant, corps étranger». Le travail de Ruiz nous apparaît lié à cette conception de la trace, qui cesse d'opposer présence et absence:

[La trace] permet de penser la présence de ce qui n'est plus ou pas encore, de ce qui ressortit à un univers autre, et de penser cette présence non sur le mode de la substitution et du subterfuge, du simulacre et de la simulation, mais sur celui de la différence, du dérangement. [...] Elle atteste le mouvement, l'acte même de passage, ce flux de moments pluriels qui préparent, à l'horizon d'une construction perceptive, l'élaboration d'un objet intentionnel. (EF, p. 17)

Cette perception «à la trace», qui caractérise toute expérience de spectature, touche plusieurs axes centraux de la réflexion de Ruiz observés précédemment: le dépassement des contradictions apparentes, l'accès au "monde autre ", la valeur du passage, la pluralité, la contamination de plusieurs modes de temps. La capacité du film de se faire "champ de mines» nous apparaît reposer sur son mode d'interprétation même, qui procède par accumulation de traces et est calqué sur la perception du sonore, même lorsqu'il s'agit de l'image visuelle.

L'exemple de l'ambiguïté des sons impossibles à figer du Film à venir prend tout son sens lorsque perçu à la lumière de cette conception de la trace:

Le son naît, résonne et meurt; événement plutôt que chose, il manifeste le mouvement d'advenue dans sa pureté même. Avant de pouvoir être reconnu et nommé comme un objet sonore (clapotis ou mélodie), le son en dit l'évanescence. Il ne s'offre à l'écoute qu'entre souvenir et attente. (EF, p. 17)

Le son que nous avons associé à la «balle de plastique » pourrait justement servir à dire l'évanescence, n'étant jamais « reconnu ou nommé comme objet sonore ». Il pourrait en ce sens fournir un autre exemple de Ruiz donnant à voir le «bricolage» : le spectateur fait appel à sa mémoire pour fournir un objet à ce son, tout en demeurant déçu, car rien ne viendra jamais confirmer ou infirmer son hypothèse. En tant qu'événement répété, ce son montre délibérément le mouvement d'advenue, là où s'ouvre le film chamanique. Par ailleurs, le recours fréquent à la répétition dans ce film, tant au plan visuel que sonore, semble participer de façon générale à en faire un film ouvert, c'est-à-dire en construction permanente, illimitée. Comme nous venons de l'observer, la perception du son implique une mémoire du «déjà entendu », analogue au « déjà vu » ou au «déjà filmé » de l'image décrit et mis en valeur par Ruiz: "Chaque trace sonore porte les marques d'anciens contextes où elle s'est fait entendre: 
l'image d'une source, sous ses diverses formes, mais aussi les réactions d'écoute autrefois suscitées.» (EF, p. 19) Le recours à la mémoire du spectateur et l'attente mènent à une pluralité de liens possibles: la perception des sons passés et l'anticipation des sons à venir «se tissent» avec l'occurrence actuelle du son.

La relation qu'entretiennent le son et l'image chez Ruiz, quoique trop complexe pour être ici analysée en profondeur, se trouve également éclairée par l'essai de Campan. Un passage du texte pourrait même servir d'ébauche pour une description de l'«oralité de l'image»:

Les traces acoustiques maintiennent l'image qu'elles portent in absentia et cette image, plurielle, construite par strates au fil des multiples ancrages, vient doubler et parasiter souvent le profil singulier que circonscrit l'image entière de leur source effective. De sorte que l'icône née de l'empreinte sonore est image en éclats, tendue vers l'unité d'une figure, qui en est le pôle d'identité, le point de fuite. Le son n'est jamais exactement à l'image de quelque chose, mais pris dans le mouvement d'approche infini d'une image dont il n'offre que des fragments épars. Son interprétation ne peut être qu'une esthétique imparfaite. (EF, p. 20)

La démarche de Ruiz se présente comme un travail autour de ce "point de fuite », où la non-concordance délibérée entre la bande sonore et la bande visuelle est liée à cette conscience de l'imperfection et à la volonté de la donner à voir discutées. L'imperfection - à la fois point de départ et point d'arrivée des films de Ruiz - est bien présente dans la réalité perceptuelle quotidienne, mais redoublée par le cinéma. Si le son et l'image ne concordent jamais que de façon partielle et imparfaite au cinéma, Ruiz accentue l'écart (ou joue sur le dérangement), ce qui permet d'être "plus près des variétés de l'expérience sensible». À cet égard, le cinéma chamanique serait celui qui vise à exploiter au maximum la richesse évocatoire du film et le caractère infini de son interprétation, que ce soit par l'image, par le son, ou par les relations entre eux. 